\title{
Child height gain is associated with consumption of animal-source foods in livestock-owning households in Western Kenya
}

\author{
Emily Mosites ${ }^{1,2, *}$, George Aol ${ }^{3}$, Elkanah Otiang ${ }^{3}$, Godfrey Bigogo ${ }^{3}$, \\ Peninah Munyua ${ }^{4}$, Joel M Montgomery ${ }^{4,5}$, Marian L Neuhouser ${ }^{2,6}$, Guy H Palmer ${ }^{1}$ and \\ Samuel $M$ Thumbi $^{1}$ \\ 'Paul G. Allen School for Global Animal Health, Washington State University, PO Box 647090, Pullman, WA \\ 99164-7090, USA: ${ }^{2}$ Department of Epidemiology, University of Washington, Seattle, WA, USA: ${ }^{3}$ Kenya Medical \\ Research Institute (KEMRI), Kisian, Kenya: ${ }^{4}$ Division of Global Health Protection, Centers for Disease Control and \\ Prevention-Kenya, Nairobi, Kenya: ${ }^{5}$ Division of Global Health Protection, Center for Global Health, Centers for \\ Disease Control and Prevention, Atlanta, GA, USA: ${ }^{6}$ Fred Hutchinson Cancer Research Center, Public Health \\ Sciences, Seattle, WA, USA
}

Submitted 22 January 2016: Final revision received 10 June 2016: Accepted 5 July 2016: First published online 12 August 2016

\begin{abstract}
Objective: To clarify the pathways between household livestock and child growth by assessing the relationships between consumption of animal-source foods (ASF) and child growth and evaluating the household livestock correlates of child consumption of ASF.

Design: We conducted a longitudinal cohort study of anthropometry and $3 \mathrm{~d}$ feeding recalls among children $<5$ years old between June 2014 and May 2015. In addition, we collected data on wealth, livestock ownership and livestock diseases in the same households. We used linear and negative binomial mixed models to evaluate the relationships between household livestock characteristics, reported consumption of ASF and child growth.

Setting: An 1800-household surveillance catchment area in Western Kenya within the structure of human and animal health surveillance systems.

Subjects: Children $(n$ 874) $<5$ years old.

Results: Among children >6 months old, reported frequency of egg and milk consumption was associated with increased monthly height gain (for each additional report of consumption over $3 \mathrm{~d}$ : adjusted $\beta(95 \% \mathrm{CI})=0.010(0.002$, $0.019) \mathrm{cm} / \mathrm{month}$ and $0.008(0.004,0.013) \mathrm{cm} /$ month, respectively). Poultry ownership was associated with higher reported frequency of egg, milk and chicken consumption (adjusted incidence rate ratio $(95 \% \mathrm{CI})=1.3(1.2,1.4)$, $1.4(1.1,1.6)$ and $1.3(1 \cdot 1,1.4)$, respectively). Some livestock diseases were associated with lower reported frequency of ASF intake (livestock digestive diseases-adjusted incidence rate ratio $(95 \% \mathrm{CI})=0.89(0.78,1.00))$.

Conclusions: Child height gain was associated with milk and egg consumption in this cohort. ASF consumption was related to both household livestock ownership and animal health.
\end{abstract}

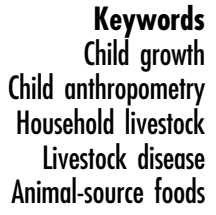

Appropriate infant and young child feeding practices and dietary intakes are necessary for optimal child growth and development. Animal-source foods (ASF) such as meat, milk and eggs are nutrient-dense and serve as excellent sources of protein, calcium, B vitamins, vitamin A and iron $^{(1)}$. As such, the use of ASF can promote diversity in the diet of young children ${ }^{(2,3)}$.

In cross-sectional surveys, ASF consumption has been associated with lower stunting prevalence ${ }^{(4)}$. Furthermore, a trial of daily provision of meat in school snacks showed improved cognitive outcomes and growth in school-aged children $^{(5)}$. Daily cow's milk supplementation during school snacks has been associated with improved B-vitamin status, also among Kenyan schoolchildren ${ }^{(6)}$. However, among pre-school children, the addition of meat or cow's milk to porridge provided at daily feeding stations over 5 months did not show a benefit for linear growth ${ }^{(7)}$. Eggs have been less commonly studied, but also have the potential to promote child growth. They provide protein and micronutrients in levels similar to other ASF, but are less expensive ${ }^{(8)}$. 
Household livestock may directly provide ASF for child consumption or provide income by which families can purchase ASF. Several studies of livestock production improvement and livestock provision have demonstrated positive impacts on ASF intake in the household ${ }^{(9)}$. However, the link between livestock ownership and feeding patterns on the child level is not clear ${ }^{(9)}$. Some studies have demonstrated that owning more household livestock is related to better anthropometric outcomes among young children ${ }^{(10)}$, while others have shown no relationship between livestock ownership and child anthropometrics ${ }^{(11,12)}$. Potentially, the complicated relationships between livestock ownership and child nutrition may be related to livestock health and wellbeing. For example, livestock that are colonized with pathogens could potentially transmit zoonotic pathogens to children ${ }^{(13,14)}$. Livestock disease could also impact child feeding patterns through loss of ASF products and income. However, the relationship between livestock disease and child feeding practices has rarely been assessed. A trial in Tanzania demonstrated that vaccinating chickens for Newcastle virus increased egg consumption among intervention households, suggesting that livestock disease might impact ASF consumption patterns ${ }^{(15)}$. Understanding the patterns and correlates of ASF consumption among rural children can provide important information to expand the use of these foods.

We previously reported that total livestock ownership in rural households was not associated with child anthropometric status, but that livestock disease was associated with some parameters of child growth ${ }^{(11)}$. In an effort to clarify the pathways involved in those findings, in the present study we evaluated three questions surrounding young child feeding practices and livestock ownership among rural households in Western Kenya. First we assessed whether the consumption of ASF was associated with child growth outcomes over time. We then evaluated whether livestock ownership or livestock disease influenced the ASF consumption patterns of these children.

\section{Methods}

\section{Study design}

The present prospective cohort study was conducted within a human and livestock health surveillance platform in Western Kenya, as described elsewhere ${ }^{(16-18)}$. During the study, population-based human health and livestock infectious disease surveillance systems were ongoing in 1800 households within a $5 \mathrm{~km}$ radius near Lake Victoria. All households enrolled in both human health and animal health surveillance systems with children who were $<5$ years of age in June 2014 were eligible for inclusion in this cohort. Caregivers provided informed consent for participation in the study. The child growth and feeding component of the study was approved by the KEMRI
Ethical Review Board as an amendment to the animal surveillance study (Scientific Steering Committee (SSC) Protocol no. 2250) and the human health surveillance study (SSC Protocol no. 1899) in March 2014.

\section{Data collection}

In May 2014, a team of community health interviewers was trained in anthropometric data collection in accordance with the World Vision training package ${ }^{(19)}$. From June 2014 to May 2015, the team visited households enrolled in the study each month to collect child feeding data and anthropometric measurements for children $<5$ years old. The team measured the length for children $<2$ years old and height for children $>2$ years old using a Shorrboard ${ }^{\circledR}$ (Weigh and Measure, LLC, Olney, MD, USA). Weight was measured using a digital standing scale (Seca, Hamburg, Germany). For infants aged $<1$ year, the child was held by his/her mother and the scale was tared to the mother's weight. Mid-upper arm circumference was assessed using a standardized tape for the purpose of screening and referral for acutely malnourished children (defined as midupper arm circumference of $<11.5 \mathrm{~cm}$ for children $>6$ months old $)^{(20)}$. Inter-observer reliability was tested as a part of initial training and team managers conducted repeat visits with a subset of children each month for quality control. If extreme height or weight values were identified in the data, the children were remeasured. These remeasurements were included in the analysis only if they were conducted within a few days of the original measurement.

Feeding patterns were assessed on a monthly basis using a structured feeding frequency questionnaire. The questionnaire was based on a previously developed household food evaluation questionnaire used in the same population $^{(18)}$. Caregivers were requested to recall the types of food and the number of times the child was fed each type of the food in the $3 \mathrm{~d}$ prior the interview. The $3 \mathrm{~d}$ recall period was determined to be preferable to a $7 \mathrm{~d}$ recall period by the study population during questionnaire piloting. Interviewers prompted feeding recall by discussing specific meals and dates with the caregivers.

Baseline livestock ownership and economic data were collected by a separate trained team through an in-depth quarterly socio-economic questionnaire between October 2013 and June 2014, as described elsewhere ${ }^{(18)}$. Interviewers asked the household head (or administrator) a series of questions regarding owned assets, owned livestock (cows, sheep, goats and chickens), income and expenditures. These data were collected on a quarterly basis.

Livestock disease information was collected between May 2014 and May 2015 by a team of veterinary technicians, also described elsewhere ${ }^{(18)}$. Briefly, human health community interviewers first requested information on whether or not there were any sick livestock in the household during a biweekly human health interview. 
The human health interviewers then reported this information to the veterinary technicians. Farmers could also report an animal disease directly through a toll-free telephone line without waiting for the biweekly visits. Upon report of a livestock disease case through either means, the veterinary technician team would evaluate the animal, make a diagnosis and provide treatment as necessary. After discussion with veterinary team leaders, cases were sub-categorized into nine syndromes including death, reproductive (abortion, stillbirths or neonatal deaths), respiratory (cough, nasal discharges, difficulty breathing), digestive (diarrhoea, bloody diarrhoea, bloating), urogenital, mammary, musculoskeletal, cutaneous and nervous system disorders. Chicken reports were restricted to cases of episodic outbreak mortality of $>30 \%$ of the flock, reflective of the most common losses in household poultry.

\section{Data analyses}

We analysed the data to evaluate three main hypotheses: (i) whether ASF consumption was associated with child height or weight velocity; (ii) whether livestock ownership was associated with increased ASF intake; and (iii) whether livestock disease was associated with decreased ASF intake. All analyses were conducted using the statistical software package Stata/SE 13. To describe the cohort overall, we calculated proportions, means, minimums and maximums of baseline child and household characteristics. For each regression analysis, associations were tested univariably and then adjusted for predetermined potential confounders or precision variables. Tests were two-sided and were considered statistically significant if the confidence interval did not include zero or one (in the case of incidence rate ratios).

\section{Analysis of animal-source food consumption and child growth}

We assessed whether the $3 \mathrm{~d}$ frequency of reported ASF intake each month was associated with child height or weight gain over time. Food items were constructed as the frequency reported over the $3 \mathrm{~d}$ recall period each month as time-varying predictors. We also categorized foods into groups based on the Food and Nutrition Technical Assistance programme recommendations ${ }^{(21)}$ (including grains and tubers; vitamin A-rich fruits; other fruits and vegetables; meat, poultry or fish; legumes; dairy; and eggs). We used the number of these categories reported to create a baseline $3 \mathrm{~d}$ dietary diversity score.

We used linear mixed models to model the outcome of child height or weight each month. The models included random intercepts for household and child to account for multiple measurements per child over time and multiple children per household. We also included a random slope for each child. The primary independent variable for this model was time, as measured by month of the child's height and weight measurement. We tested whether the reported frequency of ASF consumption was significantly associated with the effect of time on child height or weight (i.e. the child's growth) by evaluating an interaction between reported ASF and month. We used the same model specifications to explore the relationship between each non-ASF food frequency, including breast-milk intake, and child growth.

All predictors were first tested in univariable analysis, after which we adjusted for the a priori-determined factors of household wealth and income, number of household members, child baseline age and child sex. These four adjustment variables were chosen as potential confounders in the pathway between ASF consumption and child growth. The asset-based wealth score was constructed using principal component analysis from reported ownership of assets such as farming implements, bikes, vehicles, radios, tractors, telephones, motorbikes, televisions, computers, electronics, dwellings and buildings, and latrines ${ }^{(22)}$. We also used a measure of the household's quarterly off-farm income in increments of 5000 Kenyan shillings (approximately \$US 50) as an additional indicator of household socio-economic position. Because of the difference in both consumption and growth among infants under 6 months of age, these models were restricted to children who were older than 6 months at baseline. Due to the high number of comparisons between the non-ASF foods and growth, the non-ASF model results were presented non-parametrically. During data cleaning, highly outlying values within a child's growth curve were removed from analysis (representing $<0.7 \%$ of measurements).

\section{Analysis of livestock ownership and animal-source food consumption}

We evaluated whether livestock ownership was associated with reported $3 \mathrm{~d}$ frequency of ASF intake among children. Livestock ownership counts were calculated as a household count within each livestock species (chickens, cows, sheep and goats). Cow counts were used specifically, instead of total cattle, as they represent the potential milk source (goats are not a significant source of milk in this population). Because livestock ownership varies over time, we averaged these measurements across 9 months (three measurement points) for each household, before the beginning of anthropometric measurements, to provide an overall estimate of household livestock ownership.

We constructed negative binomial mixed models to evaluate the outcome of $3 \mathrm{~d}$ count of reported ASF intake. We chose these models because the distribution of each reported ASF showed evidence of overdispersion, and assessed this assumption in the final models by testing the $\alpha$ parameter using a likelihood ratio test. These models included random intercepts for child and household. The count of livestock-sourced ASF intake was constructed both as a total sum of the number of times the child ate any ASF (milk, eggs, meat or poultry meat) and the number of times each separate food was eaten over the $3 \mathrm{~d}$ period. 
After evaluating the univariable associations, we adjusted for the a priori-determined factors of child age, child sex, number of household members, household wealth, ownership of other livestock and off-farm income, as defined above. These variables were chosen as potential confounders in the pathway between household livestock ownership and child consumption patterns, or as predictors of the outcome (in the case of child age and sex). Although breast-feeding is recommended exclusively until 6 months of age, some infants $<6$ months old were fed ASF and so these models were not restricted by age. As a sensitivity analysis, we assessed whether these effects differed when the models excluded children aged $<6$ months.

\section{Analysis of livestock disease and animal-source food consumption}

We tested if livestock disease episodes were associated with reported $3 \mathrm{~d}$ frequency of ASF intake among children. Livestock disease was defined as a report of any livestock syndrome, in any species, within the household over $30 \mathrm{~d}$ periods between May 2014 and April 2015. Households could report more than one disease case per month, and disease counts were treated as a continuous variable. Digestive diseases (a category of livestock diseases) and diarrhoeal diseases (a sub-category within digestive diseases) were tested both in combination with other diseases and as separate categories, because they were the most commonly reported syndromes. Livestock diseases were linked to child feeding reports using a 1-month lag.

We tested the association between livestock disease and $3 \mathrm{~d}$ count of reported ASF intake using negative binomial mixed models with random intercepts for child and household. After assessing the associations in univariable analysis, we adjusted for the potential confounders described above. For these models, we also adjusted for total household livestock ownership as a further potential confounder. This analysis was not restricted by age for the reasons mentioned above.

\section{Results}

Eight hundred and seventy-four children (approximately $85 \%$ of the children in the catchment population) had at least one height measurement and feeding data available between June 2014 and April 2015, while 848 children had more than one data point during follow-up. Children provided a total of 7673 height, weight and feeding frequency measurement points, which represented $73 \%$ of possible height measurements.

Ninety-six per cent of the households owned at least one form of livestock. Chickens were most commonly owned ( $94.8 \%$ of households) while sheep were least commonly owned ( $25.4 \%$ of households). The distribution of livestock ownership was skewed, with a few households owning many animals while most households owned a small number. Although households owned an average of nine chickens, over one-third of the households owned fewer than five chickens, and a few households had large poultry production systems with greater than forty chickens. Household livestock also varied over the three livestock ownership measurement time points. Between October 2013 and June 2014 on average, one in three households gained or lost a single cow, one in two households gained or lost a single sheep, three-quarters of households gained or lost a single goat, and household chicken ownership changed by six birds.

Among those with a first measurement within 3 months of June 2014 (814 children), child age ranged from 1 month to 63 months (Table 1). At baseline and among children $>6$ months of age, slightly over one-quarter of children were reported to have consumed breast milk in the past $3 \mathrm{~d}$. The most commonly reported ASF was fish and the most commonly reported non-ASF was ugali (a cornmeal staple of the region). The most commonly reported livestock- and poultry-sourced ASF was eggs, followed by milk, meat and poultry meat.

Over time, caregivers described feeding patterns which varied slightly by season (Fig. 1). The highest reported frequency of total food intake was in December, while the lowest was in August. These seasonal changes were driven primarily by carotenoid-rich fruits (e.g. mangos) and other fruits and vegetables (e.g. guavas), which are seasonal in the study area. Report of an additional instance of breast milk, porridge, cow's milk, eggs and rice consumption was each significantly associated with increased height gain over time (Fig. 2). Report of increased frequency of ugali, papaya and greens was each associated with increased weight gain over time. Report of increased frequency of porridge, eggs, chicken and rice was each marginally associated with lower weight gain over time. Although fish was a commonly consumed source of protein in the region, the frequency of fish intake was not associated with height or weight gain.

Table 2 shows the estimates for consumption of specific ASF and child height/weight gain over time. Egg consumption was significantly associated with child height gain over time (each additional instance of consuming eggs over 3 d period: adjusted $\beta=0.010$ (95\% CI 0.002 , $0.019) \mathrm{cm} / \mathrm{month})$. Based on the average growth of children in the cohort, a child who ate eggs once per day during the recall period grew $5 \%$ more in height than a child who ate no eggs. Cow's milk intake was also significantly associated with child height gain over time (each additional instance over $3 \mathrm{~d}$ period: adjusted $\beta=0.008$ (95\% CI $0.004,0.013) \mathrm{cm} /$ month). A child who consumed milk an average of once per day each day grew 3\% more in height over one month than a child who did not consume any milk in this cohort. No ASF were associated with increased weight gain among children, although eggs were marginally associated with lower weight gain. The dietary diversity score was 
Table 1 Child and household characteristics at baseline, Asembo, Western Kenya, 2014-2015 ( $n$ 814)

\begin{tabular}{|c|c|c|c|c|c|c|}
\hline Characteristic & $n$ & $\%$ & Mean & SD & Minimum & Maximum \\
\hline \multicolumn{7}{|l|}{ Child characteristics } \\
\hline Age (months) & - & - & $34 \cdot 1$ & $16 \cdot 6$ & $1 \cdot 2$ & $63 \cdot 3$ \\
\hline Sex, female & 431 & $52 \cdot 9$ & - & - & - & - \\
\hline \multicolumn{7}{|l|}{$3 \mathrm{~d}$ recall of foods eaten at baseline* } \\
\hline Dietary diversity (no. of food groups) & - & - & 4.6 & 1.4 & 1 & 7 \\
\hline Breast milk & 222 & $25 \cdot 4$ & 2.5 & $5 \cdot 4$ & 0 & 30 \\
\hline Cow's milk & 97 & $11 \cdot 1$ & 0.65 & 0.25 & 0 & 30 \\
\hline Eggs & 140 & $16 \cdot 0$ & 0.30 & 0.61 & 0 & 3 \\
\hline Meat & 111 & $12 \cdot 7$ & 0.22 & 0.54 & 0 & 4 \\
\hline Chicken & 37 & $4 \cdot 2$ & 0.08 & 0.34 & 0 & 3 \\
\hline Fish & 666 & $76 \cdot 2$ & 1.9 & 1.6 & 0 & 16 \\
\hline Ugali (cornmeal staple) & 606 & $69 \cdot 3$ & 4.0 & 3.3 & 0 & 18 \\
\hline \multicolumn{7}{|l|}{ Household characteristics } \\
\hline Household members (count) & - & - & 5.9 & $2 \cdot 0$ & 2 & 13 \\
\hline Off-farm income $†$ (\$US $\ddagger)$ & 572 & 75.4 & 110 & 164 & 0 & 1224 \\
\hline \multicolumn{7}{|l|}{ Livestock owned§ (count) } \\
\hline Cattle & 445 & 53.5 & $2 \cdot 1$ & $4 \cdot 1$ & 0 & 51 \\
\hline Chickens & 829 & 94.8 & $9 \cdot 1$ & $7 \cdot 8$ & 0 & 57 \\
\hline Goats & 418 & 47.8 & 1.6 & $2 \cdot 8$ & 0 & 22 \\
\hline Sheep & 222 & 25.4 & 1.0 & $2 \cdot 9$ & 0 & 23 \\
\hline
\end{tabular}

*Percentage reporting that the child consumed any of the food items in the recall period (yes/no); mean, sD, minimum, maximum frequency of times eaten over the $3 \mathrm{~d}$ recall period; among children aged 6-59 months.

†Percentage reporting any income; mean, SD, minimum, maximum of three-quarter average of reported off-farm household income.

$\ddagger \$ \cup S 1 \approx 100$ Kenyan shillings.

$\S$ Percentage owning any of the livestock species; mean, SD, minimum, maximum of three-quarter average of household livestock owned.

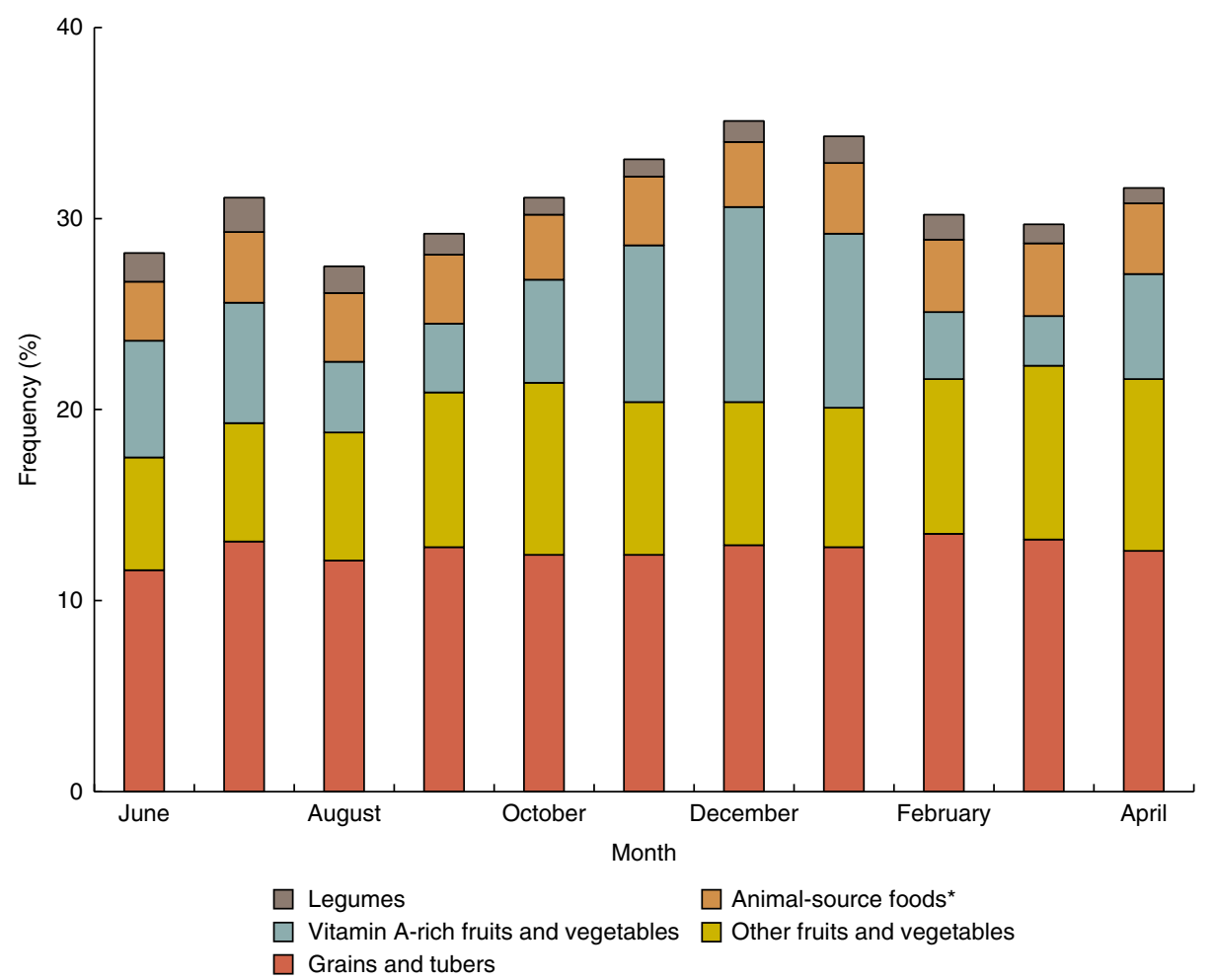

Fig. 1 Reported frequency of food groups eaten among children in Asembo, Western Kenya, by month, 2014-2015. *Comprised of fish, eggs, cow's milk, meat and chicken

marginally associated with height gain, but not associated with weight gain.

Ownership of specific livestock species was associated with $3 \mathrm{~d}$ report of ASF intake (Table 3). Adjusting for ownership of other animals, wealth, income, child age and child sex, increased poultry ownership was associated with increased consumption of eggs, milk and chicken meat. Each additional ten chickens owned was associated with a $30 \%$ increased reported frequency of egg intake, a $40 \%$ increased reported frequency of milk intake and 


\begin{tabular}{|l|l|l|}
\hline Food item & Height gain & Weight gain \\
\hline Breast milk & & \\
\hline Porridge & & \\
\hline Cow's milk & & \\
\hline Eggs & & \\
\hline Rice & & \\
\hline Ugali (cornmeal) & & \\
\hline Papaya & & \\
\hline Greens & & \\
\hline Chicken & & \\
\hline Guava & & \\
\hline Mango & & \\
\hline Wheat products & & \\
\hline Fish & & \\
\hline Beans/legumes & & \\
\hline Avocado & & \\
\hline Oranges & & \\
\hline Tomato & & \\
\hline Potato & & \\
\hline Bananas & & \\
\hline Meat & & \\
\hline Cabbage & & \\
\hline
\end{tabular}

\begin{tabular}{|l|l|}
\hline \multicolumn{2}{|c|}{ Association with growth ${ }^{\star}$} \\
\cline { 1 - 1 }$P<0.01$ & \multirow{2}{*}{ Negative effect size } \\
\cline { 1 - 1 }$P<0.1$ & \\
\hline$P \geq 0.1$ & Neutral effect size \\
\hline$P<0.1$ & \multirow{2}{*}{ Positive effect size } \\
\cline { 1 - 1 }$P<0.01$ & \\
\hline
\end{tabular}

Fig. 2 Reported consumption of individual food items in the prior month and subsequent height/weight gain over time among children >6 months of age, Asembo, Western Kenya, 2014-2015. *As tested by linear mixed models of time-varying food consumption and child height or weight gain over time

Table 2 Linear mixed models of frequency of dietary diversity, animal-source food (ASF) intake and subsequent growth among children $>6$ months of age, Asembo, Western Kenya, 2014-2015

\begin{tabular}{|c|c|c|c|c|c|}
\hline Food intake* & Primary outcome & Unadjusted $\beta$ (n 848) & $95 \% \mathrm{Cl}$ & Adjusted $\beta$ (n 735) & $95 \% \mathrm{Cl}$ \\
\hline \multirow[t]{2}{*}{ Dietary diversity scoreł } & Height gain (cm/month) & 0.008 & $0.002,0.014$ & 0.006 & $0.000,0.013$ \\
\hline & Weight gain (kg/month) & 0.003 & $-0.002,0.007$ & 0.002 & $-0.003,0.007$ \\
\hline \multirow[t]{2}{*}{ All ASF } & Height gain (cm/month) & 0.005 & $0.002,0.008$ & 0.005 & $0.002,0.009$ \\
\hline & Weight gain ( $\mathrm{kg} /$ month) & -0.000 & $-0.003,0.002$ & -0.001 & $-0.003,0.002$ \\
\hline \multirow[t]{2}{*}{ Eggs } & Height gain (cm/month) & 0.011 & $0.003,0.019$ & 0.010 & $0.002,0.019$ \\
\hline & Weight gain ( $\mathrm{kg} /$ month) & -0.006 & $-0.012,0.000$ & -0.007 & $-0.014,0.000$ \\
\hline \multirow[t]{2}{*}{ Cow's milk } & Height gain ( $\mathrm{cm} /$ month) & 0.007 & $0.003,0.011$ & 0.008 & $0.004,0.013$ \\
\hline & Weight gain (kg/month) & 0.001 & $-0.002,0.004$ & 0.006 & $-0.003,0.004$ \\
\hline \multirow[t]{2}{*}{ Meat } & Height gain (cm/month) & 0.002 & $-0.006,0.010$ & 0.002 & $-0.006,0.011$ \\
\hline & Weight gain (kg/month) & -0.002 & $-0.008,0.004$ & -0.002 & $-0.009,0.004$ \\
\hline \multirow[t]{2}{*}{ Chicken } & Height gain (cm/month) & 0.007 & $-0.005,0.019$ & 0.007 & $-0.006,0.020$ \\
\hline & Weight gain (kg/month) & -0.007 & $-0.017,0.003$ & -0.009 & $-0.020,0.001$ \\
\hline
\end{tabular}

Significant results are indicated in bold font.

†Adjusted for child sex, child age at baseline, household asset-based wealth and number of household members.

†Estimate for one additional food group consumed over the recall period.

a $30 \%$ increased reported frequency of chicken meat intake. Each additional cow owned was associated with a $13 \%$ increase in reported frequency of milk consumption, although this association was not significant. These results did not meaningfully differ when we excluded children $<6$ months of age.

Overall, livestock diseases tended to be associated with decreased reported frequency of total ASF consumption among children in the following month, although these associations were not significant (see online supplementary material, Table S1). Among individual ASF items, some livestock disease cases were significantly associated with reported frequency of consumption in the following month (Table 4). Controlling for household livestock ownership, household asset-based wealth, household income and child age, livestock digestive disease among 
Table 3 Negative binomial mixed model of specific livestock ownership and child feeding between June 2014 and May 2015, Asembo, Western Kenya ( $n$ 758)

\begin{tabular}{|c|c|c|c|c|c|c|c|c|}
\hline \multirow[b]{3}{*}{ Household livestock ownership $†$} & \multicolumn{8}{|c|}{ Child $3 \mathrm{~d}$ feeding frequency, adjusted ${ }^{*}$} \\
\hline & \multicolumn{2}{|c|}{ Egg intake } & \multicolumn{2}{|c|}{ Cow's milk intake } & \multicolumn{2}{|c|}{ Meat intake } & \multicolumn{2}{|c|}{ Chicken intake } \\
\hline & IRR & $95 \% \mathrm{Cl}$ & IRR & $95 \% \mathrm{Cl}$ & IRR & $95 \% \mathrm{Cl}$ & IRR & $95 \% \mathrm{Cl}$ \\
\hline Poultry ownership & 1.03 & $1.02,1.04$ & 1.04 & $1.01,1.06$ & 1.00 & $0.99,1.01$ & 1.03 & $1.01,1.04$ \\
\hline Cow ownership & 1.05 & $1.00,1.11$ & $1 \cdot 13$ & $0.98,1.31$ & 1.00 & $0.93,1.08$ & 1.05 & $0.98,1.13$ \\
\hline Sheep ownership & 1.00 & $0.97,1.02$ & 0.93 & $0.87,0.99$ & 1.00 & $0.97,1.03$ & 1.01 & $0.97,1.05$ \\
\hline Goat ownership & 0.99 & $0.96,1.02$ & 1.01 & $0.94,1.08$ & 1.01 & $0.98,1.03$ & 1.00 & $0.96,1.03$ \\
\hline
\end{tabular}

$\mathrm{IRR}$, incidence rate ratio.

Significant results are indicated in bold font.

${ }^{*}$ Adjusted for all species ownership categories, household asset wealth, off-farm income, number of household members, child sex and child age.

†Average count over three financial quarters between October 2013 and June 2014.

Table 4 Negative binomial mixed models of time-varying livestock disease and child feeding in the following month, Asembo, Western Kenya, 2014-2015 (n 758)

\begin{tabular}{|c|c|c|c|c|c|c|c|c|}
\hline \multirow[b]{3}{*}{ Livestock disease } & \multicolumn{8}{|c|}{ Child $3 d$ feeding frequency, adjusted ${ }^{*}$} \\
\hline & \multicolumn{2}{|c|}{ Egg intake } & \multicolumn{2}{|c|}{ Cow's milk intake } & \multicolumn{2}{|c|}{ Meat intake } & \multicolumn{2}{|c|}{ Chicken intake } \\
\hline & IRR & $95 \% \mathrm{Cl}$ & IRR & $95 \% \mathrm{Cl}$ & IRR & $95 \% \mathrm{Cl}$ & IRR & $95 \% \mathrm{Cl}$ \\
\hline Any sick livestock $†$ & 0.99 & $0.96,1.03$ & 0.88 & $0.71,1.09$ & 0.98 & $0.90,1.06$ & 1.00 & $0.97,1.03$ \\
\hline Any livestock with digestive diseaseł & 0.81 & $0.69,0.96$ & 0.89 & $0.62,1.28$ & 0.88 & $0.71,1.10$ & 0.90 & $0.66,1.22$ \\
\hline Any livestock with diarrheal disease & 1.04 & $0.85,1.28$ & 0.99 & $0.62,1.58$ & 0.69 & $0.50,0.97$ & 0.66 & $0.41,1.05$ \\
\hline Cattle cases $\S$ & 0.93 & $0.79,1.11$ & 1.27 & $0.89,1.83$ & 1.04 & $0.83,1.30$ & 0.82 & $0.58,1.16$ \\
\hline Sheep cases§ & 1.06 & $0.90,1.26$ & 0.54 & $0.29,0.99$ & 1.00 & $0.75,1.32$ & 1.08 & $0.74,1.58$ \\
\hline Goat cases $\S$ & 0.89 & $0.73,1.08$ & 0.82 & $0.52,1.29$ & 1.02 & $0.79,1.31$ & 0.95 & $0.66,1.35$ \\
\hline Chicken death & 1.04 & $0.96,1.12$ & 0.58 & $0.25,1.35$ & 0.90 & $0.73,1.12$ & $1 \cdot 15$ & $1.02,1.28$ \\
\hline
\end{tabular}

IRR, incidence rate ratio.

Significant results are indicated in bold font.

*Adjusted for total livestock owned, household wealth, household income, number of household members and child age.

†Cases of disease or death among cattle, sheep and goats; cases of flock death among chickens.

¥Among cattle, sheep and goats.

$\S$ Cases of death or disease.

cattle, sheep or goats was associated with a $19 \%$ decrease in the reported frequency of child intake of eggs. Livestock diarrhoeal disease report in the prior month was associated with a $31 \%$ decrease in reported frequency of meat consumption. Death or disease in sheep was associated with a $46 \%$ decrease in reported frequency of milk consumption. However, a report of chicken flock death in the prior month was associated with a $15 \%$ increase in the report of the frequency of children eating chicken.

The livestock and demographic predictors of dietary diversity are included in the online supplementary material, Table S2. Ownership of poultry, household wealth and off-farm income were significantly related to higher numbers of food groups consumed across the follow-up period (adjusted $\beta=0.015$ (95\% CI 0.01, 0.02), adjusted $\beta=0.08$ (95\% CI 0.04, 0.11) and adjusted $\beta=0.03$ (95\% CI 0.01, 0.04), respectively). Higher numbers of household members and season were inversely associated with dietary diversity (adjusted $\beta=-0.03$ (95\% CI -0.05 , -0.01 ) and adjusted $\beta=-0.02$ (95\% CI $-0.02,-0.01$ ), respectively). Report of any livestock disease was marginally associated with lower dietary diversity (adjusted $\beta=-0.008(95 \% \mathrm{CI}-0 \cdot 02,0 \cdot 00))$.

\section{Discussion}

In this cohort of children $<5$ years old in rural Western Kenya, we evaluated the relationships between ASF consumption and growth and then assessed the drivers of ASF consumption. Although our previous analyses have not shown significant relationships between livestock ownership and child anthropometric status or growth ${ }^{(11,12)}$, here we found that higher frequency of consumption of ASF, particularly milk and eggs, was associated with larger gains in child height, and that ASF intake was associated with livestock ownership. These findings indicate that there is a possibility for household livestock to be beneficial for child growth, but that there may be additional barriers to a direct benefit. Livestock disease may be one of these barriers, suggesting that livestock health interventions may help optimize the translation between livestock ownership and child growth.

\section{Eggs}

At baseline, approximately $16 \%$ of children were reported to have consumed eggs in the prior $3 \mathrm{~d}$. 
Reported frequency of egg intake throughout follow-up was associated with increasing monthly height gains in this cohort, even after controlling for age and other potential confounders. This finding aligns with previous literature, which finds that eggs provide protein and micronutrients that could promote child growth ${ }^{(11)}$. However, we also found that eggs were marginally associated with decreased weight gain. This may be a result of multiple comparisons or confounding factors. The primary household characteristics that were associated with egg consumption were the number of chickens owned and the average off-farm income of the household. We found that certain livestock digestive diseases significantly predicted decreased egg consumption, despite these diseases being reported only among cattle, goats and sheep. These diseases, reported among other livestock, might not directly impact chicken health and production, but rather may represent a loss of wealth in the household. These losses may lead to the sale of poultry or eggs, which could result in fewer poultry products available for child consumption. This finding indicates that the pathways between household livestock health, child dietary intake and child growth are complex. Little research has been conducted in this area and further study is necessary. Qualitative and mixed methods studies can provide insight into perplexing health and socio-economic household pathways such as these.

\section{Cow's milk}

Frequency of cow's milk consumption was also associated with height gain over time among children in this cohort, also after adjusting for age and other potential confounders. However, only $11 \%$ of children were reported to have consumed milk at baseline and this proportion decreased to $7 \%$ by the end of follow-up. Cow's milk was frequently consumed among young children (1-2 years of age) and appears to be a weaning food in this population. Milk consumption was predicted by household chicken ownership. Chicken production may be related to milk intake through the sale of poultry and eggs to purchase cow's milk, particularly among families that do not own cows or do not have a currently lactating cow. Cow ownership also showed a trend towards increasing reported milk intake frequency among children. Livestock disease generally had a negative association with reported child milk consumption, although most of these associations were not significant.

\section{Poultry and other meat}

Poultry meat and other meat consumption were not associated with monthly child height or weight gain. Consumption of poultry meat was uncommon ( $4 \%$ at baseline) and was slightly associated with lower weight gain compared with children who did not eat chicken. Household poultry ownership, poultry flock death and season were associated with higher reported chicken consumption. This likely reflects consumption of sick and dead chickens, which can still be valued as a food source despite zoonotic disease transmission risks. Consumption of other meats was more common than chicken meat consumption ( $13 \%$ at baseline) and was not associated with livestock ownership. Meat consumption was primarily associated with off-farm income. Other studies have found that meat consumption was associated with improved growth parameters ${ }^{(23)}$. It is possible that we did not see this benefit due to the low frequency or small quantities of meat being consumed in this population. Fish was consumed at a high frequency among the children in this study. However, we did not identify an association between fish consumption and linear or ponderal growth, which may be a result of the low variability in consumption patterns.

\section{Study strengths and limitations}

The present study had several strengths, including its longitudinal design and thorough data collection for household factors such as livestock ownership, household wealth and income, and livestock disease. However, it is observational in nature which provides potential for reverse causality and confounding in the associations between child diet and growth. For example, increasing frequency of eating guava was associated with lower height gain among children. While it is possible that eating guava at high frequencies does not comprise a sufficient diet for growth, it may also represent a seasonal lack of availability of other foods. Furthermore, child age is tightly linked to both children's diet and their growth trajectory. Residual confounding by age may lead to associations between diet and growth that are in fact age-related. Also, although we collected frequency data for each food item, we did not collect information regarding the quantities of foods eaten. Because the livestock ownership data were collected by a different team at a different frequency, we used ownership information that was collected prior to the collection of feeding information to create representative household averages. We expect that the primary disadvantage to evaluating previous livestock ownership with nondifferential variability within households would be a nullifying effect on the size of the effect between livestock ownership and feeding practices. Although most children were available for most visits, some height, weight and feeding frequency measurements were missing, which comprises another limitation. Finally, livestock disease reports were low in number on the household level, which is illustrated by the wider confidence intervals that the livestock disease estimates provided. These limitations necessitate a nuanced interpretation of the results.

\section{Conclusion}

In addition to infectious disease prevention, maternal nutrition promotion and other nutrition-sensitive 
interventions, young children require a diverse, high-quality diet for ideal growth and development. Our findings suggest that the promotion of egg and milk consumption can support linear growth among children, and that both the ownership and the health of household livestock can influence child feeding practices. However, small proportions (4-16\%) of children were reported to have consumed ASF from cows, goats, sheep or chickens throughout follow-up, representing an important opportunity for nutrition promotion in rural families. Additionally, given the complex relationships between livestock ownership, livestock disease and child feeding, qualitative approaches could facilitate the understanding of feeding choices within households. Interventions that promote the pathway between household livestock ownership and child dietary intake could provide nutritional benefit among rural children.

\section{Acknowledgements}

Financial support: This work was conducted with funds from the Paul G. Allen Family Foundation and the National Institutes of Health post-doctoral training fellowship (grant number T32 AI07025). The funders had no role in the design, analysis or writing of this article. Conflict of interest: None. Authorship: E.M. and S.M.T. contributed to the design, fieldwork, analysis and writing of the manuscript. G.A., E.O. and G.B. coordinated the fieldwork. P.M., J.M.M., M.L.N. and G.H.P. contributed to the design and writing of the manuscript. Ethics of buman subject participation: This study was conducted according to the guidelines laid down in the Declaration of Helsinki and all procedures involving human subjects/patients were approved by the KEMRI Ethical Review Board. Written or verbal informed consent was obtained from all subjects/patients. Disclaimer: The findings and conclusions in this report are those of the authors and do not necessarily represent the official position of the Centers for Disease Control and Prevention.

\section{Supplementary material}

To view supplementary material for this article, please visit http://dx.doi.org/10.1017/S136898001600210X

\section{References}

1. Allen LH \& Dror DK (2011) Effects of animal source foods, with emphasis on milk, in the diet of children in low-income countries. Nestle Nutr Workshop Ser Paediatr Program 67, $113-130$.

2. Mallard SR, Houghton LA, Filteau S et al. (2014) Dietary diversity at 6 months of age is associated with subsequent growth and mediates the effect of maternal education on infant growth in urban Zambia. J Nutr 144, 1818-1825.

3. Pan American Health Ogranization, World Health Organization (2003) Guiding Principles for Complementary
Feeding of the Breastfed Child. Washington, DC: Division of Health Promotion and Protection, PAHO.

4. Krebs NF, Mazariegos M, Tshefu A et al. (2011) Meat consumption is associated with less stunting among toddlers in four diverse low-income settings. Food Nutr Bull 32, 185-191.

5. Neumann CG, Bwibo NO, Murphy SP et al. (2003) Animal source foods improve dietary quality, micronutrient status, growth and cognitive function in Kenyan school children: background, study design and baseline findings. J Nutr $\mathbf{1 3 3}$, 11 Suppl. 2, 3941S-3949S.

6. Siekmann JH, Allen LH, Bwibo NO et al. (2003) Kenyan school children have multiple micronutrient deficiencies, but increased plasma vitamin B-12 is the only detectable micronutrient response to meat or milk supplementation. J Nutr 133, 11 Suppl. 2, 3972S-3980S.

7. Long JK, Murphy SP, Weiss RE et al. (2012) Meat and milk intakes and toddler growth: a comparison feeding intervention of animal-source foods in rural Kenya. Public Health Nutr 15, 1100-1107.

8. Iannotti LL, Lutter CK, Bunn DA et al. (2014) Eggs: the uncracked potential for improving maternal and young child nutrition among the world's poor. Nutr Rev $\mathbf{7 2}$, 355-368.

9. Azzari C, Cross E, Haile B et al. (2014) Does Livestock Ownership Affect Animal Source Foods Consumption and Child Nutritional Status? Washington, DC: World Bank Group.

10. Jin M \& Iannotti LL (2014) Livestock production, animal source food intake, and young child growth: the role of gender for ensuring nutrition impacts. Soc Sci Med 105, $16-21$.

11. Mosites E, Thumbi SM, Otiang E et al. (2016) Relations between household livestock ownership, livestock disease, and young child growth. J Nutr 146, 1118-1124.

12. Mosites EM, Rabinowitz PM, Thumbi SM et al. (2015) The relationship between livestock ownership and child stunting in three countries in Eastern Africa using national survey data. PLoS One 10, e0136686.

13. Kagira JM \& Kanyari PW (2010) Occurrence of risk factors for zoonoses in Kisumu City, Kenya: a questionnaire survey. East Afr J Public Health 7, 1-4.

14. Marquis GS, Ventura G, Gilman RH et al. (1990) Fecal contamination of shanty town toddlers in households with non-corralled poultry, Lima, Peru. Am J Public Health 80, 146-149.

15. Knueppel D, Cardona C, Msoffe P et al. (2010) Impact of vaccination against chicken Newcastle disease on food intake and food security in rural households in Tanzania. Food Nutr Bull 31, 436-445.

16. Breiman RF, Van Beneden CA \& Farnon EC (2013) Surveillance for respiratory infections in low- and middleincome countries: experience from the Centers for Disease Control and Prevention's Global Disease Detection International Emerging Infections Program. J Infect Dis 208, Suppl. 3, S167-S172.

17. Odhiambo FO, Laserson KF, Sewe M et al. (2012) Profile: the KEMRI/CDC Health and Demographic Surveillance System - Western Kenya. Int J Epidemiol 41, 977-987.

18. Thumbi SM, Njenga MK, Marsh TL et al. (2015) Linking human health and livestock health: a 'one-health' platform for integrated analysis of human health, livestock health, and economic welfare in livestock dependent communities. PLoS One 10, e0120761.

19. Nutrition Centre of Expertise, World Vision International, Global Health \& WASH (2012) Measuring and Promoting Child Growth: Facilitator's Manual. Ontario: World Vision International. 
20. World Health Organization \& UNICEF (2009) WHO Child Growth Standards and the Identification of Severe Acute Malnutrition in Infants and Children. Geneva: WHO.

21. Kennedy G, Ballard T \& Dop M (2013) Guidelines for Measuring Household and Individual Dietary Diversity. Rome: Nutrition and Consumer Protection Division, FAO.
22. Vyas S \& Kumaranayake L (2006) Constructing socioeconomic status indices: how to use principal components analysis. Health Policy Plan 21, 459-468.

23. Neumann CG, Murphy SP, Gewa C et al. (2007) Meat supplementation improves growth, cognitive, and behavioral outcomes in Kenyan children. J Nutr 137, 1119-1123. 